\title{
Ambient Temperature Effects on Evaluation of Plant Physiological Activity Using Plant Bioelectric Potential
}

\author{
Yuki Hasegawa*, Genta Yamanaka, Ki Ando ${ }^{1}$ and Hidekazu Uchida \\ Saitama University, 255 Shimo-okubo, Sakura-ku, Saitama 338-8570, Japan \\ ${ }^{1}$ Tokyo Denki University, 5 Senju Asahi-cho, Adachi-ku, Tokyo 120-8551, Japan
}

(Received March 3, 2014; accepted May 1, 2014)

Key words: plant bioelectric potential, plant factory, photosynthesis, ambient temperature, plant physiological activity

The plant factory has been in practical use for ensuring a stable food supply and cultivating high-quality food. However, it has problems with capital and operating expenditures, because it is necessary to prepare a big room of the same level of cleanliness as that of a semiconductor factory and to use a full environment control system that includes an air conditioner, a feed nutrient solution line, and sensing devices in continuous operation. Therefore, we focused on the bioelectric potential response as a promising and low-cost approach of evaluating plant physiological activities. Bioelectric potential is generated by ions in a plant cell and the potential is related to plant physiological activities. Although we already reported that the potential response differs depending on the on-off cycle of illumination and is related to photosynthetic activity, there is no report on the effects of ambient temperature and light interruption time on the measurement of bioelectric potential. In this study, we investigated the effects of ambient temperature and light interruption time on the evaluation of plant physiological activities. In the experiments, we measured bioelectric potential responses and $\mathrm{CO}_{2}$ concentration when light irradiation was started or stopped at several temperatures. In addition, we investigated the effect of the light interruption time on the evaluation of plant physiological activities using plant bioelectric potential. These results contribute to the improvement of the precision of the evaluation system for plant physiological activities using bioelectric potential responses.

*Corresponding author: e-mail: yuki@ees.saitama-u.ac.jp 


\section{Introduction}

Recently, it has been a global issue of securing safe and abundant food. The plant factory has been in practical use for ensuring a stable food supply and cultivating highquality food. In the plant factory, a system controls artificial light, temperature, humidity, water including nutrition, and carbon dioxide concentration to create an optimal cultivation environment in an indoor space, and does not use pesticides. ${ }^{(1-3)}$ Thus, the essential characteristic of the plant factory is not only productivity but also the ability to produce a stable amount of clean and safe food all year round.

However, the plant factory has problems with capital and operating expenditures, because it needs to maintain a stable and full environment control system that includes an air conditioner, a feed nutrient solution line, and sensing devices. Therefore, the important issues are cost reduction and productivity of the improvement. ${ }^{(4,5)}$ Various studies have been carried out to investigate how to reduce these costs and improve the productivity of cultivation. ${ }^{(6-8)}$

In our previous work, we focused on the measurement of bioelectric potential as a low-cost technique of evaluating plant physiological activities, such as photosynthesis, respiration, and transpiration, and supporting effective cultivation. The bioelectric potential of a plant is generated by ions inside the plant cells. ${ }^{(9)}$ Measurement of the plant bioelectric potential is a promising method of real-time evaluation and monitoring of the plant's state of activity as a physical quantity. We reported that the bioelectric potential varies when the illumination is started or stopped, that the amplitude of this variation is correlated with the photosynthetic rate, and that the characteristics of the bioelectric potential depend on the illumination period, wavelength, and intensity. ${ }^{(10-12)}$ Although we also showed that the potential response differs depending on the illumination conditions, there is little discussion on the effect of ambient temperature on the behavior of the potential response. To improve the photosynthetic rate and productivity in a plant factory, it is desirable that the light interruption time is as short as possible. However, in our present evaluation method, light has to be interrupted for at least $30 \mathrm{~min}$ to observe a significant potential response when the illumination is started. Thus, the photosynthetic reaction is reasonably disturbed during light interruption time. Therefore, it is necessary to study the shortest optimal light interruption time for the evaluation of using the bioelectric potential response.

In this study, we aim to clarify the effect of ambient temperature on the evaluation of plant activity using plant bioelectric potential. The optimal temperature differs depending on the plant species, and the cultivation temperature affects the photosynthetic rate. Several studies have been carried out on the temperature dependence of the photosynthetic rate and other physiological activities of plants. ${ }^{(13-15)}$ In our experiments, the bioelectric potential response under light irradiation and interruption was observed at several cultivation temperatures. We also observed the bioelectric potential response when the light interruption time was gradually shortened. These results contribute to the improvement of the precision of the evaluation system for plant physiological activities using bioelectric potential responses. 


\section{Materials and Methods}

\subsection{Measurements of plant bioelectric potential and photosynthetic rate}

Figure 1 shows the measurement system of the plant bioelectric potential. The plant was placed in a $22.4 \mathrm{~L}$ closed vessel, and the change in $\mathrm{CO}_{2}$ concentration caused by plant metabolic processes, such as photosynthesis and respiration, was measured using a nondispersive infrared (NDIR)-type $\mathrm{CO}_{2}$ analyzer (LI-840, Meiwafosis Co., Ltd.). The ambient temperature and humidity were controlled by the growth chamber (BAC-130H, Espec Corp.) enclosing the vessel. The measurement temperature was controlled at every $5{ }^{\circ} \mathrm{C}$ from 15 to $35{ }^{\circ} \mathrm{C}$ if necessary, and the humidity was controlled at 60 to $70 \%$ RH. The light source consisted of blue $(470 \mathrm{~nm})$, green $(525 \mathrm{~nm})$, red $(660 \mathrm{~nm})$, and infrared $(735 \mathrm{~nm})$ LEDs, and light irradiation patterns were controlled by a control unit. The photosynthetically active photon flux density (PPFD) on the leaf surface was 150 $\mu \mathrm{mol} \cdot \mathrm{m}^{2} \cdot \mathrm{s}^{-1}$.

The sample plants were some golden pothos plants (Epipremunum aureum) grown in hydroponics in $0.5 \mathrm{~L}$ plastic pots and have two to three leaflets. The bioelectric potential was measured using electroencephalographic (EEG) disk-type electrodes of $9 \mathrm{~mm}$ diameter (NE-155A, Nihon Kohden Corp.) attached to the plant surface with a conductive paste for EEG (Z-401CE, Nihon Kohden Corp.). To detect the potential response induced by photosynthetic reactions, an electrode was attached to the leafstalk as a reference electrode and another electrode was attached to the leaf surface, where photosynthesis takes place. The potential difference between the two electrodes was measured with a high-input-impedance ( $>1 \mathrm{G} \Omega$ ) digital multimeter (DMM in Fig. 1, R6552A, ADC Corp.) and recorded by a computer at a sampling interval of $1 \mathrm{~s}$. The measurement was started $60 \mathrm{~min}$ after the electrode attachment because of the exclusion of a noise signal generated immediately after electrode attachment.

To determine the photosynthetic rate, an NDIR-type $\mathrm{CO}_{2}$ analyzer (LI-840, Meiwafosis Co., Ltd.) was used. First, we measured the increase in $\mathrm{CO}_{2}$ concentration caused by the respiration of the plant in the dark, and defined it as the respiration rate ( $\mathrm{ppm} / \mathrm{h}$ ). Then, we determined the sum of the observed decrease rate in $\mathrm{CO}_{2}$ concentration owing

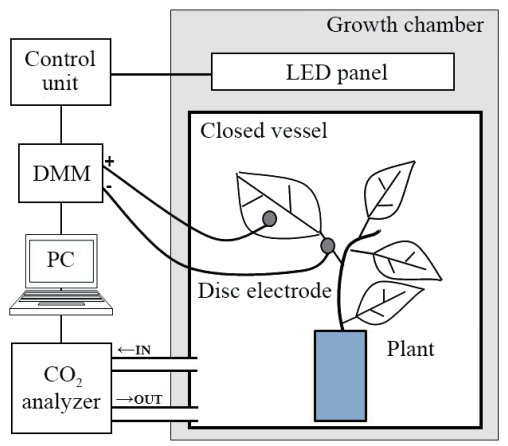

Fig. 1. (Color online) Measurement system of bioelectric potential. 
to photosynthetic activity under illumination and the respiration rate, and defined it as the photosynthetic rate $(\mathrm{ppm} / \mathrm{h})$.

\subsection{Evaluation parameters and measurement conditions}

We measured the bioelectric potential response of the sample plant to a stimulus of light irradiation or interruption. Figure 2 shows the typical bioelectric potential responses when illumination was started and stopped. The potential rose immediately after light irradiation and passed a peak, and slowly returned to approximately the initial value. On the other hand, when the illumination was stopped, the potential dropped sharply, and then rose toward the steady state. We defined the difference $V_{\text {on }}$ [Fig. 2(a)] as that between the largest potential increase and the value before light irradiation, and the difference $V_{\text {off }}$ [Fig. 2(b)] as that between the largest potential decrease and the value before light interruption. In a previous study, it was reported that the amplitude of the potential variation $V_{\text {on }}$ obtained by starting the illumination has a strong correlation with the photosynthetic rate, and that the $V_{\text {off }}$ obtained by stopping the illumination has a strong correlation with photochemical reactions and the respiration rate. These results suggested that these parameters can be used to evaluate the various physiological activities of the plant. ${ }^{(11,12)}$

In our experiments, first, we investigated the temperature dependence of bioelectric potential response and photosynthetic rate. The bioelectric potential and photosynthetic rate were measured from 15 to $35^{\circ} \mathrm{C}$ at $5{ }^{\circ} \mathrm{C}$ intervals (the optimal temperature of golden pothos plants is about $20^{\circ} \mathrm{C}$ ). Generally, the photosynthesis-temperature curve is often symmetrical or bell-shaped. ${ }^{(15,16)}$

Next, we also observed the bioelectric potential response when the light interruption time was gradually shortened. The light irradiation time was fixed at $1 \mathrm{~h}$, and only the light interruption time was changed $(1,3,5$, and $10 \mathrm{~min})$.

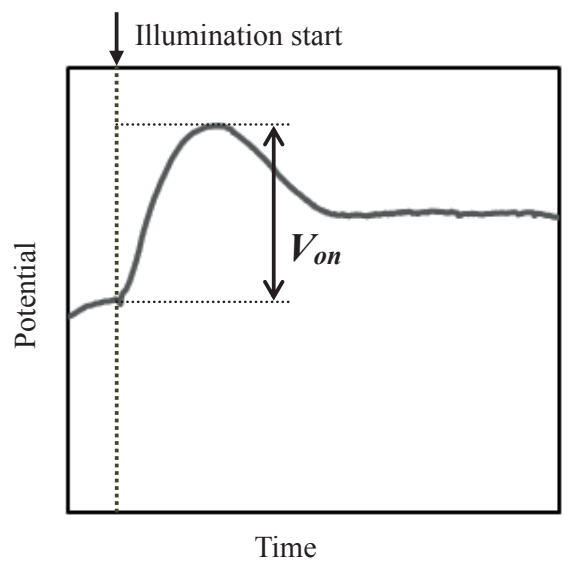

(a)

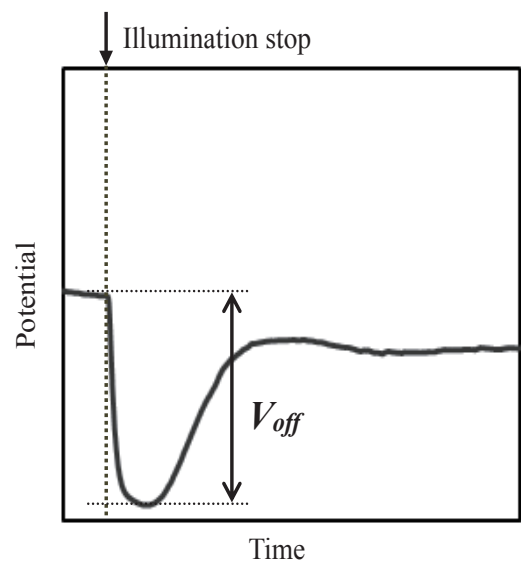

(b)

Fig. 2. Typical bioelectric potential response to (a) light irradiation and (b) light interruption and definition of evaluation parameters $V_{\text {on }}$ and $V_{\text {off }}$. 


\section{Results and Discussion}

\subsection{Relationship between ambient temperature and bioelectric potential response}

First, we compared the characteristics of the bioelectric potential responses at various ambient temperatures. Figure 3 shows the time series data of bioelectric potential responses from 15 to $35{ }^{\circ} \mathrm{C}$ at $5{ }^{\circ} \mathrm{C}$ intervals, when the illumination was started and stopped repeatedly. In this figure, the potential responses changed immediately with light irradiation and interruption at all ambient temperatures. The waveform of the potential response differs depending on the ambient temperature, and the characteristics

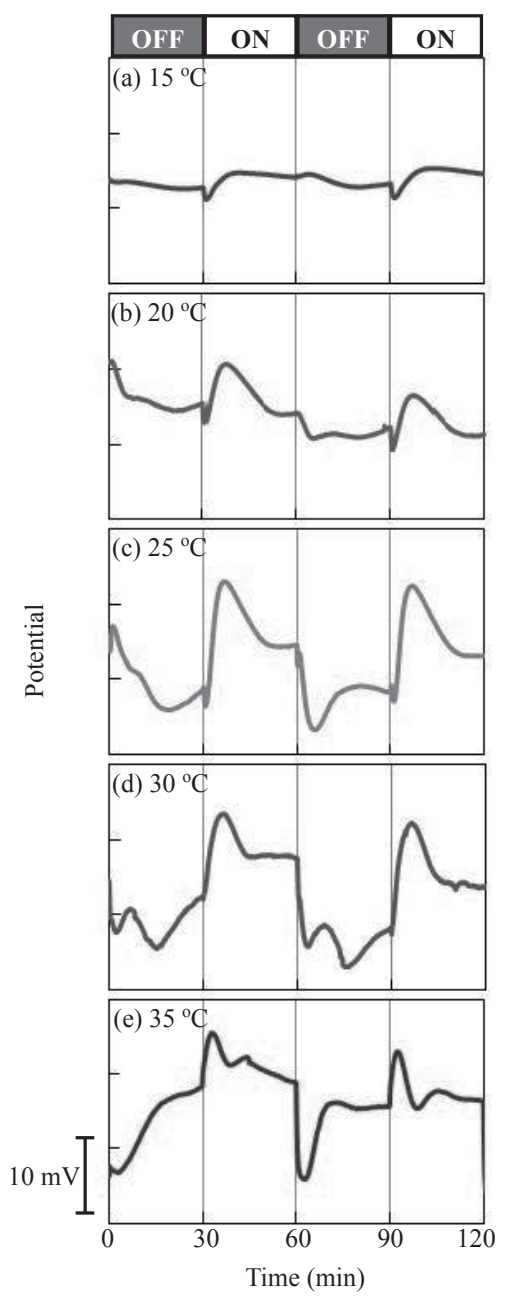

Fig. 3. Time series data of bioelectric potential response from 15 to $35{ }^{\circ} \mathrm{C}$ at $5{ }^{\circ} \mathrm{C}$ intervals. 
of potential increases and decreases are reproducible at every temperature. In particular, the amplitudes of the potential at 25 and $30{ }^{\circ} \mathrm{C}$ showed the largest responses, and that at $15{ }^{\circ} \mathrm{C}$ showed the smallest response when the illumination was started. On the other hand, the amplitudes of the potential gradually increased from a low temperature to a high temperature when the illumination was stopped.

We also measured the change in $\mathrm{CO}_{2}$ concentration in response to light irradiation and interruption, and the relationship between the ambient temperature and photosynthetic rate of the sample plants is shown in Fig. 4. This figure shows that the photosynthetic rate differed with the ambient temperature. In this figure, the plotted marks are the mean value from each set of eight data points, and each error bar indicates the standard deviation. It was also shown that the photosynthesis-temperature curve had a peak between 25 and $30{ }^{\circ} \mathrm{C}$. Generally, the optimal temperature of golden pothos plants is about $20{ }^{\circ} \mathrm{C}$, but it was reported that the optimal temperature changes depending on the growth temperature. Plants grown at low temperatures show greater photosynthetic capabilities at lower temperatures, whereas plants grown at high temperatures show greater photosynthetic capabilities at higher temperatures. ${ }^{(17)}$ Thus, it is thought that the optimal temperature of the sample plant was changed to $20{ }^{\circ} \mathrm{C}$ by our growth environment.

Figure 5 shows $V_{\text {on }}$ (a) and $V_{\text {off }}$ (b) at various ambient temperatures. In this figure, the plotted marks are the mean value from each set of eight data points, and each error bar indicates the standard deviation (at only $15{ }^{\circ} \mathrm{C}$, the mean value and standard deviation were calculated from five data points, because the potential variation was sometimes too small to read). It was found from Fig. 5(a) that each $V_{\text {on }}$ data is variable but tends to show a bell shape similarly to the photosynthesis-temperature curve (Fig. 4), and that the peak temperature is between 25 and $30{ }^{\circ} \mathrm{C}$. Figure $5(\mathrm{~b})$ indicates that $V_{\text {off }}$ tended to increase with ambient temperature. According to plant physiological studies, the dark

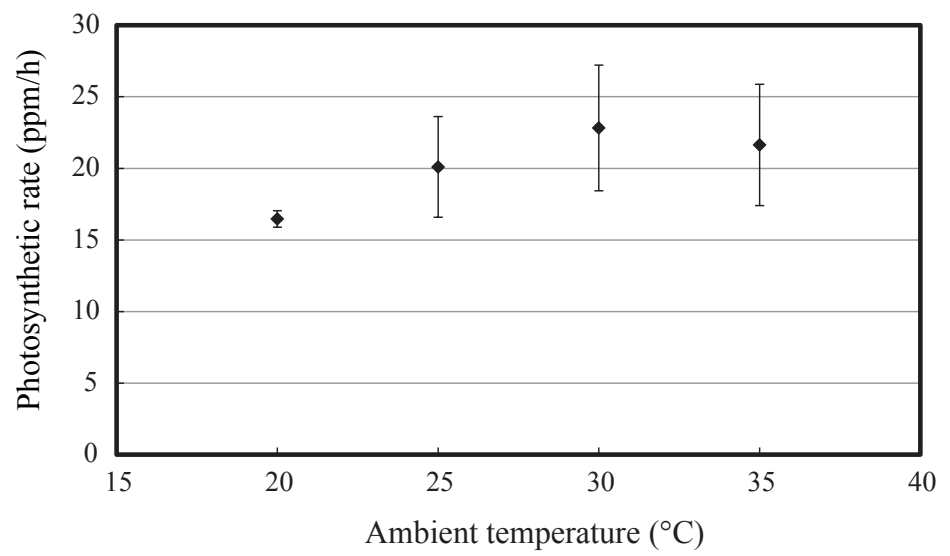

Fig. 4. Photosynthetic rates of sample plants at various ambient temperatures. 


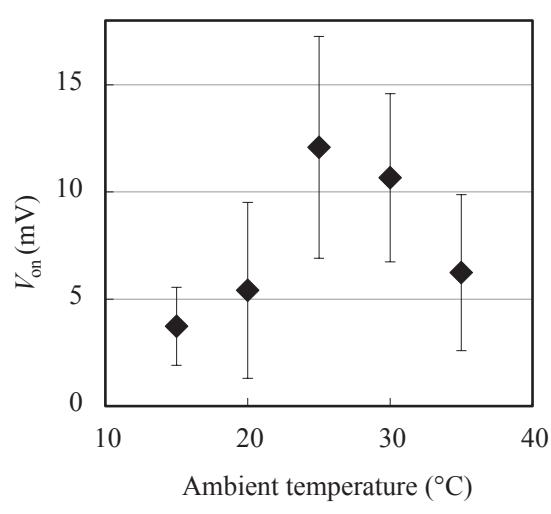

(a)

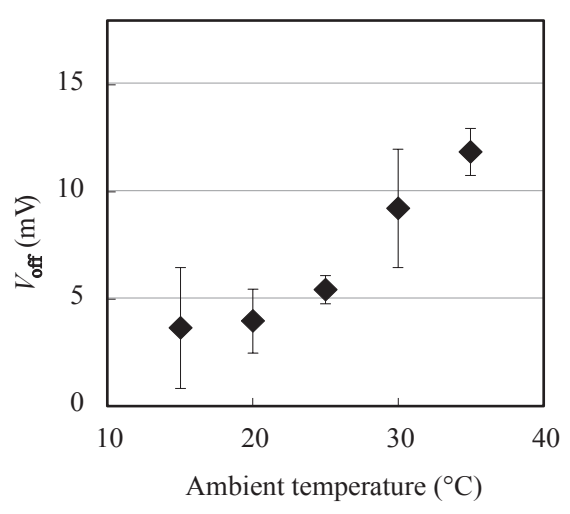

(b)

Fig. 5. (a) $V_{\text {on }}$ and (b) $V_{\text {off }}$ at various ambient temperatures.

respiration and chloroplast electron transport rates increased with increasing ambient temperature. ${ }^{(14,16,17)}$ In contrast, the activation state of the plant enzyme, ribulose-1,5bisphosphate carboxylase/oxygenase (RuBisCO), decreased with increasing ambient temperature. RuBisCO is the enzyme involved in the Calvin cycle that catalyzes the first major step of carbon fixation. ${ }^{(13)}$ It was considered that the observed $V_{\text {off }}$ was related to these plant physiological activities. Therefore, a more detailed investigation of the relationship between the plant physiological activities and the bioelectric potential responses when light illumination was stopped is required in our future work.

\subsection{Light interruption time and bioelectric potential responses}

To improve the photosynthetic rate and productivity in a plant factory, it is desirable that the light interruption time is as short as possible. However, in our present evaluation method, light has to be interrupted for at least $30 \mathrm{~min}$ to observe a significant potential response when the illumination is started. The photosynthetic reaction is reasonably disturbed during light interruption time. Therefore, it is necessary to study the shortest optimal light interruption time for the evaluation of plant physiological activities using the bioelectric potential response.

In this section, we observed the bioelectric potential response when the light interruption time was gradually shortened. Figure 6 shows the bioelectric potential responses at various light interruption times. We observed very simple responses when the interruption time was $1 \mathrm{~min}$. The potential response decreased immediately after light interruption was observed, and then the potential increased rapidly up to the initial values after light illumination. The potential responses at the interruption times of 3 and $10 \mathrm{~min}$ had similar waveforms. First, the potential responses decreased and had the first small peak about $1 \mathrm{~min}$ after the light interruption. Then, the response at $3 \mathrm{~min}$ decreased gradually until the light illumination was started, and the response at $10 \mathrm{~min}$ also decreased until the second peak was formed and then increased gradually until the light 


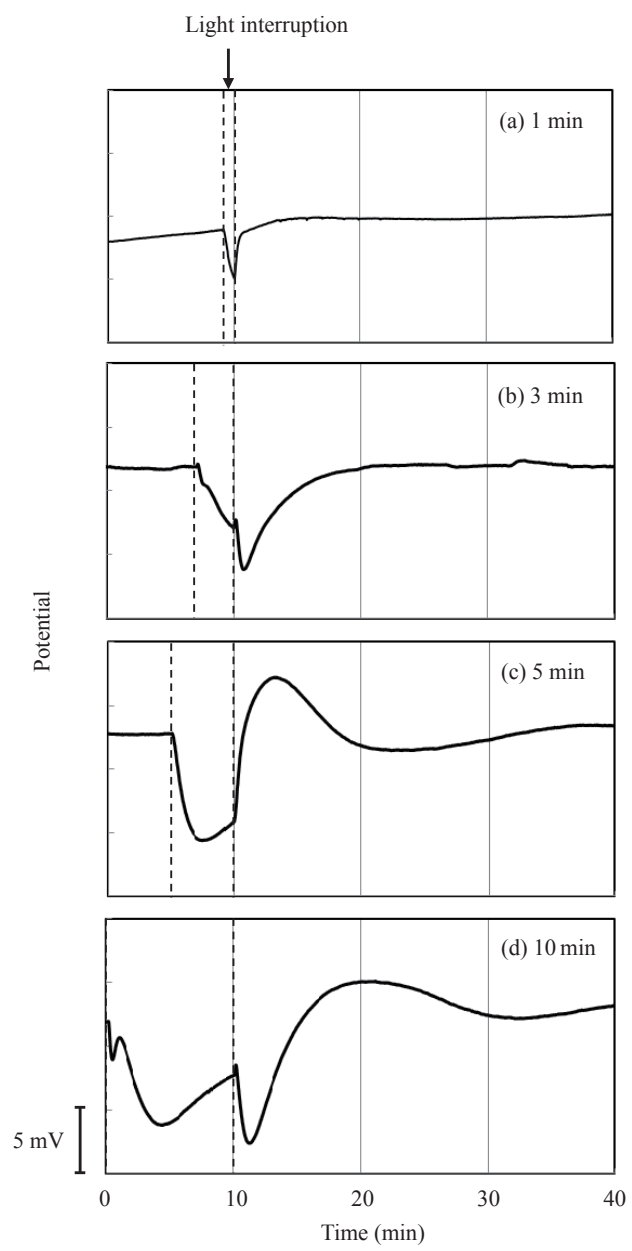

Fig. 6. Bioelectric potential responses at various light interruption times.

illumination was started. After the light illumination was started, the potential slightly increased, and then decreased soon and had a peak. Finally, we observed the distinctive potential response when the interruption time was $5 \mathrm{~min}$. The potential response did not include several small peaks; thus, it looks like a sine curve. All waveform trends were observed repeatedly in multiple plants. We obtained the difference in potential response depending on the light interruption time. We also required the measurement of the $\mathrm{CO}_{2}$ concentration and the analysis of the relationships between plant physiological activities and potential responses in these experiments. 


\section{Conclusions}

In this study, we investigated the effects of ambient temperature and light interruption time on the evaluation of plant activities. We measured the potential difference of the two electrodes on the leaflet and leafstalk of the sample plant. To determine the photosynthetic rate, an NDIR-type $\mathrm{CO}_{2}$ analyzer was used. The measurement temperature was controlled from 15 to $35{ }^{\circ} \mathrm{C}$ at $5{ }^{\circ} \mathrm{C}$ intervals when necessary, and the humidity was controlled at 60 to $70 \% \mathrm{RH}$ using the growth chamber. First, the temperature dependence of the bioelectric potential responses was investigated at various ambient temperatures. The obtained results showed that the potential responses changed immediately with light irradiation and interruption at all ambient temperatures. We also indicated that plant physiological activities, such as the photosynthetic and respiration rates, could be expressed by $V_{\text {on }}$ and $V_{\text {off }}$. Therefore, a more detailed investigation of the relationship between plant physiological activities and bioelectric potential responses when light illumination is started and stopped should be carried out in our future work. Finally, we also observed the bioelectric potential response when the light interruption time was gradually shortened $(1,3,5$, and $10 \mathrm{~min})$. The obtained results showed that the difference in potential response depended on the light interruption time. These results contribute to the improvement of the precision of the evaluation system for plant physiological activities using bioelectric potential responses.

\section{Acknowledgements}

This work was supported by a JSPS Grant-in-Aid for Young Scientists (A), Grant No. 24686049 .

\section{References}

1 T. Morimoto, T. Torii and Y. Hashimoto: Control Eng. Pract. 3 (1995) 505.

2 I. Ioslovich and P. Gutman: Automatica 36 (2000) 1665.

$3 \mathrm{M}$. Hu, Y. Chen and L. Huang: Int. J. Production Economics (in press).

4 M. Takatsuji: Fundamentals and Practices of Plant Factories (Shokabo, Toyko, 2007) (in Japanese).

5 M. Takatsuji: J. Soc. High Technol. Agric. 22 (2010) 2 (in Japanese).

6 M. Nishimura, T. Kozai, C. Kubota and C. Chun: J. Soc. High Technol. Agric. 13 (2001) 204 (in Japanese).

7 Y. Miyoshi, K. Hidaka, T. Okayasu, O. Hirano, D. Yasutake and M. Kitano: Env. Cont. in boilo. 51 (2013) 89

8 W. Yamori: J. Plant Biochem. Pysiol. 1 (2013) 113.

9 M. Tazawa, T. Shinmen and T. Mimura: Inst. Electrostat. Jpn. 6 (1982) 285 (in Japanese).

10 K Ando, Y. Hasegawa, H. Maekawa and T. Katsube: IEICE Trans. Electron. E91-C (2008) 1905.

11 K. Ando, Y. Hasegawa, T. Yaji and H. Uchida: Elec. and Com. in Jpn. 95 (2012) 10.

12 K. Ando, Y. Hasegawa, T. Yaji and H. Uchida: Elec. and Com. in Jpn. 96 (2013) 85.

13 W. Yamori, K. Suzuki, K. Noguchi, M. Nakai and I. Terashima: Plant, Cell \& Environ. 29 (2006) 1659. 
14 W. Yamori, K. Noguchi and I. Terashima: Plant, Cell \& Environ. 28 (2005) 536.

15 W. Yamori, J. R. Evans and S. Caemmerer: Plant, Cell \& Environ. 33 (2010) 332.

16 L. Taiz and E. Zeiger: Plant physiology, 5th ed. (Sinauer Associates, Inc., Sunderland, 2010) 255.

17 W. Yamori, K. Hirosaka and D. A. Way: Photosynth. Res. 119 (2014) 101.

\section{About the Authors}

Yuki Hasegawa received her Master of Education and her Doctor Degree in Engineering from Saitama University in 2001 and 2005, respectively. She is now an associate professor at the Department of Electrical and Electronics Systems, Saitama University, since 2014. Her current research interests are environmental monitoring and controlling systems using plant bioelectric potential, and taste sensors using several types of sensing films. She is a member of the Institute of Electrical Engineers of Japan, the Japan Society of Applied Physics, and the Japanese Society of Agricultural, Biological and Environmental Engineers and Scientists.

Genta Yamanaka received his B. Eng. Degree from Saitama University in 2013. He is a master course student of the Division of Mathematics, Electronics and Informatics, Graduate School of Science and Engineering, Saitama University. $\mathrm{He}$ is a student member of the Institute of Electrical Engineers of Japan.

Ki Ando received his D. Eng. Degree from Graduate School of Science and Engineering, Saitama University, in 2012. Since 2013, he is an assistant professor at Faculty of Engineering of Tokyo Denki University. His research interests are plant bioelectric potential responses to light and their applications. His research field is electronic measurement technology enhancing the quality of human life. He is a member of the Institute of Electrical Engineers of Japan and the Research Institute of Signal Processing Japan.

Uchida Hidekazu received his Master Degree in Electronics Engineering and his Doctor Degree in Engineering from Saitama University in 1990 and 1997, respectively. He is a professor at the Department of Electrical and Electronic Systems, Saitama University, since 2014. He is a member of the Japan Society of Applied Physics and the Institute of Electrical Engineers of Japan. 